\title{
Charge carrier dynamics and self-trapping on $\mathrm{Sb}_{2} \mathbf{S}_{3}(100)$
}

\author{
Lisa Grad $\odot,{ }^{1}$ Fabian O. von Rohr, ${ }^{2}$ Matthias Hengsberger ${ }^{\circ},{ }^{1}$ and Jürg Osterwalder ${ }^{1}{ }^{1, *}$ \\ ${ }^{1}$ Department of Physics, University of Zurich, Winterthurerstrasse 190, 8057 Zurich, Switzerland \\ ${ }^{2}$ Department of Chemistry, University of Zurich, Winterthurerstrasse 190, 8057 Zurich, Switzerland
}

(Received 15 March 2021; accepted 15 June 2021; published 8 July 2021; corrected 28 July 2022)

\begin{abstract}
Antimony sulfide $\left(\mathrm{Sb}_{2} \mathrm{~S}_{3}\right)$ is a promising material for solar energy conversion. It consists of earth-abundant elements with a low toxicity and high stability. Furthermore, it shows suitable optical and electronic properties like a large absorption coefficient and a convenient band gap of $1.7 \mathrm{eV}$. Nevertheless, so far, the obtained power conversion efficiencies and the open circuit voltages are far below the theoretical limits and need to be increased for a viable application of $\mathrm{Sb}_{2} \mathrm{~S}_{3}$-based cells at the large scale. To achieve this it is important to identify the dominating loss mechanism. Here time-resolved two-photon photoemission experiments are presented from the (100) surface of a cleaved $\mathrm{Sb}_{2} \mathrm{~S}_{3}$ single-crystal to investigate the charge carrier dynamics after photoexcitation. Based on these measurements an ultrafast relaxation within below 150 fs towards the conduction band minimum is observed. This is followed by a decay within $1 \mathrm{ps}$ into states, which are located in the band gap $0.06 \mathrm{eV}$ and $0.44 \mathrm{eV}$ below the conduction band minimum where the charge carriers have long lifetimes of $27 \mathrm{ps}$ and $63 \mathrm{ps,}$ respectively. Based on the energetic position, the energy width and the formation time of the lower-lying gap state a self-trapping mechanism of free charge carriers by optical phonons with a frequency of $1.44 \mathrm{THz}$ is proposed, and the results are discussed in this context. These findings provide evidence that the poor performance of $\mathrm{Sb}_{2} \mathrm{~S}_{3}$ in solar devices can be traced back to intrinsically formed traps that can hardly be avoided.
\end{abstract}

DOI: 10.1103/PhysRevMaterials.5.075401

\section{INTRODUCTION}

The conversion of solar energy in high-efficiency lowcost photovoltaic or photoelectrochemical solar cells that can be built from stable, abundant, and nontoxic materials is a desirable approach for a sustainable energy production at the large scale. So far, however, solar cells still show low power conversion efficiencies (PCE) at high fabrication costs. When investigating suitable low-cost systems that deliver high PCE, interest in antimony-based chalcogenides like antimony sulfide $\left(\mathrm{Sb}_{2} \mathrm{~S}_{3}\right)$ or antimony selenide $\left(\mathrm{Sb}_{2} \mathrm{Se}_{3}\right)$ arose. Both materials show a high stability, consist of earthabundant elements, and can be synthesized at comparatively low temperatures. Due to the high absorption coefficients and favorable band gaps of $1.7 \mathrm{eV}\left(\mathrm{Sb}_{2} \mathrm{~S}_{3}\right)$ and $1.1 \mathrm{eV}$ $\left(\mathrm{Sb}_{2} \mathrm{Se}_{3}\right)$, they are excellent absorbers for solar light. The crystal structure is strongly anisotropic with covalently bonded one-dimensional (1D) ribbons that are in turn bonded to each other by much weaker van der Waals forces [1,2]. Owing to this ribbon structure the bonds at the grain boundaries are saturated which reduces recombination losses [3]. The crystal structure together with the orthorhombic unit cell and the Brillouin zone of $\mathrm{Sb}_{2} \mathrm{~S}_{3}$ are shown in Fig. 1.

\footnotetext{
*Corresponding author: osterwal@physik.uzh.ch

Published by the American Physical Society under the terms of the Creative Commons Attribution 4.0 International license. Further distribution of this work must maintain attribution to the author(s) and the published article's title, journal citation, and DOI.
}

So far, most attempts to employ $\mathrm{Sb}_{2} \mathrm{~S}_{3}$ for solar energy conversion were aimed at thin film solar cells, but there were also approaches for the application as front subcell material for silicon-based tandem solar cells [4] or as electrode in photoelectrochemical cells $[5,6]$. For the subcell material in tandem cells it was shown that the optimum band gap is $1.7-1.8 \mathrm{eV}$. This matches with the values reported for $\mathrm{Sb}_{2} \mathrm{~S}_{3}$ and leads to a theoretical PCE of 40\% [7]. In case of thin-film solar cells the highest efficiencies reported so far are $7.5 \%$ [8] for nanostructured and 6.6\% [9] for planar cells. Those are far below the theoretical value in the Shockley-Queisser limit of $28.6 \%$ [10]. Furthermore, the highest obtained open circuit voltages are $0.7 \mathrm{~V}$ for both types, which is only half of the theoretical value. The similar characteristics of both cell types suggest that the dominating losses occur in the bulk of $\mathrm{Sb}_{2} \mathrm{~S}_{3}$. To identify the main loss mechanism, the charge carrier dynamics after photoexcitation are important. By absorption of light, electron-hole pairs are generated, which need to be separated and transferred to the opposite electrode surfaces from where they can be extracted to an external circuit or an electrolyte for instance. Thereby, the efficiency is limited by fast charge carrier recombination, trapping in bulk and surface defect states or by a sluggish charge transfer towards and across the interfaces.

However, up to now the charge carrier dynamics in $\mathrm{Sb}_{2} \mathrm{~S}_{3}$ are poorly investigated and most studies were performed on heterostructures [11,12]. In Ref. [12], poor charge carrier transport properties were reported based on transient absorption spectroscopy measurements in combination with carrier diffusion modeling. It was shown that holes in $\mathrm{Sb}_{2} \mathrm{~S}_{3}$ based solid-state semiconductor-sensitized solar cells are first 
(a)

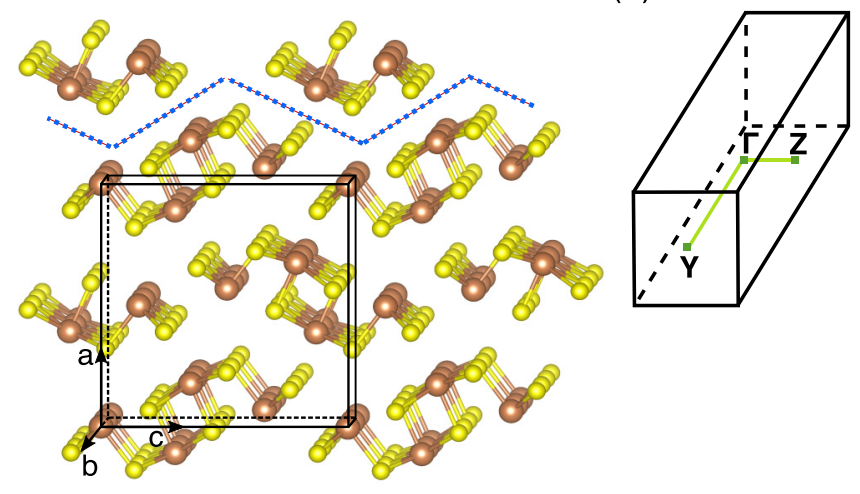

FIG. 1. Crystal structure of antimony sulfide. (a) Crystal structure of antimony sulfide $\left(\mathrm{Sb}_{2} \mathrm{~S}_{3}\right)$ with the ribbons pointing along $\vec{b}$. The $\mathrm{Sb}$ atoms are colored in brown and the $\mathrm{S}$ atoms in lemon. The cleavage plane (blue dashed line) and the orthorhombic unit cell (black line) are marked. (b) Brillouin zone with the high-symmetry directions along $(\Gamma \mathrm{Y})$ and across ribbons $(\Gamma \mathrm{Z})$ indicated.

trapped in deep trap levels in the bulk causing a slow bulkto-surface transport. Moreover, low hole injection rates into an adjacent hole conduction layer were observed. The authors suggested sulfur vacancies as the origin of those deep trap states, but the defect structure of $\mathrm{Sb}_{2} \mathrm{~S}_{3}$ is still not fully understood. In addition, the pristine material was studied by Yang and coworkers [13]. There, time-resolved photoluminescence measurements on poly- and single-crystalline samples are presented which were grown by different methods to vary the concentration and composition of the intrinsic defects. Based on photoabsorption measurements the authors could confirm the value for the band gap of $1.7 \mathrm{eV}$, in agreement with other values reported so far. In photoluminescence a Stokes shift of $0.6 \mathrm{eV}$ was observed and was attributed to trap emission. To clarify the origin of those trap states the intrinsic defect concentrations were determined for all growth methods and rather low values in the order of $10^{10} \mathrm{~cm}^{-3}$ were obtained. In contrast to that, fluence-dependent photoluminescence measurements showed that the density of emitting trap states is above $10^{20} \mathrm{~cm}^{-3}$ and thus exceeds the intrinsic defect concentration by far. The authors concluded that the related trap states need to be formed by the $\mathrm{Sb}_{2} \mathrm{~S}_{3}$ lattice itself and they propose a polaronic self-trapping mechanism, where first the holes are trapped by phonons as a consequence of local displacements of the lattice atoms forming polarons. In a second step the trapped hole captures an electron and forms a self-trapped exciton. Nevertheless, there is still the need for further investigations of this self-trapping mechanism and the dynamics of free and trapped charge carriers.

Time-resolved two-photon photoemission (tr-2PPE) is an appropriate tool to investigate charge carrier dynamics and the trapping mechanism in $\mathrm{Sb}_{2} \mathrm{~S}_{3}$ with energy and momentum resolution. Measurements were performed on the (100) surface of a cleaved $\mathrm{Sb}_{2} \mathrm{~S}_{3}$ single crystal. By absorption of a 3-eV pump pulse electrons are excited from the valence band (VB) to the conduction band (CB) where the population and the relaxation pathways are monitored as a function of time delay on the femto to picosecond timescale. Owing to the surface sensitivity of this method the role of surface defects can be investigated. In addition, the measured signal is a superposition of electrons excited in the near-surface region and the transport of electrons in the surface depletion region due to band bending at the solid-vacuum interface [14]. In these measurements a decay of photoexcited electrons towards the conduction band minimum (CBM) within less than 150 fs is observed for $\mathrm{Sb}_{2} \mathrm{~S}_{3}$. There are two states in the band gap $0.06 \mathrm{eV}$ and $0.44 \mathrm{eV}$ below the CBM. Photoexcited electrons in the CBM decay within 1.3 ps into these gap states, where much longer lifetimes of 27 ps and 63 ps were determined, respectively. Based on a detailed analysis of the transient electron populations in CB and gap states a model for the underlying decay processes is proposed invoking free and self-trapped charge carriers.

\section{EXPERIMENTAL METHODS}

\section{A. Sample growth}

Large single crystals of $\mathrm{Sb}_{2} \mathrm{~S}_{3}$ were grown by a modified Bridgeman method. Elemental Sb (ingot, purity 99.9999\%) and S (pieces, purity $99.9995 \%$ ) were mixed in a stoichiometric ratio and sealed in a quartz ampoule under $1 / 3$ argon atmosphere. The reactants were heated to $650^{\circ} \mathrm{C}$ over the course of 12 hours, annealed at this temperature for 24 hours, then slowly cooled over 100 hours to $200^{\circ} \mathrm{C}$, and eventually quenched to room temperature. The cell parameters and phase purity of the product was checked by means of powder X-ray diffraction using a STOE STADI P diffractometer in transmission mode equipped with a Ge-monochromator using $\mathrm{Cu} \mathrm{K}_{\alpha 1}$ radiation (see Supplemental Material [15]).

\section{B. Experimental setup}

All measurements were conducted at room temperature in ultra-high vacuum (UHV) at a base pressure below $5 \times$ $10^{-10}$ mbar. Due to the small probing depth of photoemission experiments clean and well-defined surfaces are required. This was achieved by cleaving the single-crystalline samples in situ, using adhesive tape, between (100) planes, which are only bonded by weak van der Waals forces. By controlling the residues on the tape it was ensured that a closed layer was removed.

The tr-2PPE measurements were conducted using a commercial mode-locked Ti:sapphire oscillator (Coherent Mira Seed) with a regenerative pulse amplifier (Coherent RegA9050). For the measurements the repetition rate was set to $100 \mathrm{kHz}$ and the wavelength to $825 \mathrm{~nm}$ or $1.5 \mathrm{eV}$. The emitted ultrashort laser pulses with a pulse energy of $6 \mu \mathrm{J}$ and a pulse duration of $56 \mathrm{fs}$ full width at half maximum were frequency doubled in a $\beta$-barium borate (BBO) crystal to $413 \mathrm{~nm}$ or $3.0 \mathrm{eV}$. The second harmonic beam was split into a pump and a probe beam using a beam splitter. While the pump beam was guided over a computer-controlled delay stage the probe beam was again frequency doubled to $207 \mathrm{~nm}(6.0 \mathrm{eV})$ and the group velocity dispersion was compensated in $\mathrm{a} \mathrm{CaF}_{2}$ prism compressor. The polarization of each beam is adjustable using $\lambda / 2$ waveplates. Finally, the beams are superimposed on a dichroic mirror and focused collinearly onto the sample surface in the UHV system. The $1 / \mathrm{e}^{2}$ diameter of the pump 
beam on the surface was determined on a fluorescent yttrium aluminum garnet (YAG) sample with a charge-coupled device (CCD) camera to be $425 \mu \mathrm{m}$. For the measurement pump fluences below $38 \mu \mathrm{J} / \mathrm{cm}^{2}$ were used corresponding to an excitation density below $6.25 \times 10^{18} \mathrm{~cm}^{-3}$ for an absorption coefficient of $8 \times 10^{4} \mathrm{~cm}^{-1}$ of the $3-\mathrm{eV}$ light [16]. The photoelectrons were detected in a commercial hemispherical electron analyzer (Specs Phoibos 100) with resolution of energy and emission angle using a two-dimensional detector. The nondispersive axis of the two-dimensional detector allows for an acceptance angle of $\pm 15^{\circ}$ [17]. With this setup, the energy resolution is below $65 \mathrm{meV}$. A bias voltage of $-5 \mathrm{~V}$ was applied to the sample with respect to the analyzer and the position of the Fermi level was determined on a silver sample.

The valence band spectrum was measured with a monochromatic high harmonic generation (HHG) light source [18] at a photon energy of $15.3 \mathrm{eV}$. The wavelength of the laser was set to $810 \mathrm{~nm}(1.53 \mathrm{eV})$ at a repetition rate of $100 \mathrm{kHz}$. At first the fundamental was frequency-doubled in a BBO crystal to $405 \mathrm{~nm}$ and then it was focused into an argon gas jet where the fifth harmonic of the driving laser frequency is produced. The fundamental $405-\mathrm{nm}$ beam is blocked by a $200-\mathrm{nm}$-thick indium filter and the divergent $15.3-\mathrm{eV}$ beam is collimated and focused onto the sample by a spherical and a toroidal mirror pair. The HHG and the beam path towards the sample are in UHV at a base pressure below $5 \times 10^{-9}$ mbar because light at wavelengths below about $200 \mathrm{~nm}$ is absorbed in air. The $1 / \mathrm{e}^{2}$ beam waist of the HHG spot on the surface was determined on the fluorescent YAG sample with a CCD camera as $220 \mu \mathrm{m}$ which is smaller than the $\mathrm{Sb}_{2} \mathrm{~S}_{3}$ sample size.

\section{RESULTS}

\section{A. Electronic valence band structure}

Ultraviolet photoelectron spectroscopy (UPS) measurements were carried out to investigate the electronic valence band structure of a cleaved $\mathrm{Sb}_{2} \mathrm{~S}_{3}$ single crystal at the (100) surface using the HHG light source at a photon energy of $E_{P h}=15.3 \mathrm{eV}$. The light was p-polarized with respect to the plane of incidence. In Fig. 2 the measured spectrum at $\bar{\Gamma}$ near the valence band maximum (VBM) is shown. The VB onset was determined by linear extrapolation of the falling edge as $E_{\mathrm{VBM}}-E_{F}=(-1.06 \pm 0.10) \mathrm{eV}$, in good agreement with the value recently published in Ref. [5] based on X-ray photoelectron spectroscopy (XPS) measurements. Due to the higher surface sensitivity of UPS in comparison to XPS the consistency of both values is an evidence for a negligible band bending. Moreover, from the center position of the rising edge of the secondary electron cutoff the work function was determined as $\Phi=(4.55 \pm 0.15) \mathrm{eV}$. This value is in agreement with the work function which is reported in Ref. [5] based on Kelvin-probe force microscopy measurements.

\section{B. Charge carrier dynamics}

Furthermore, the $\mathrm{CB}$ electronic structure and the dynamics of charge carriers after photoexcitation at $\bar{\Gamma}$ were investigated by means of time-resolved two-photon photoemission (tr-2PPE) measurements. The principle of tr-2PPE is shown in Fig. 3(a). By the absorption of a $3 \mathrm{eV}$ pump pulse electrons

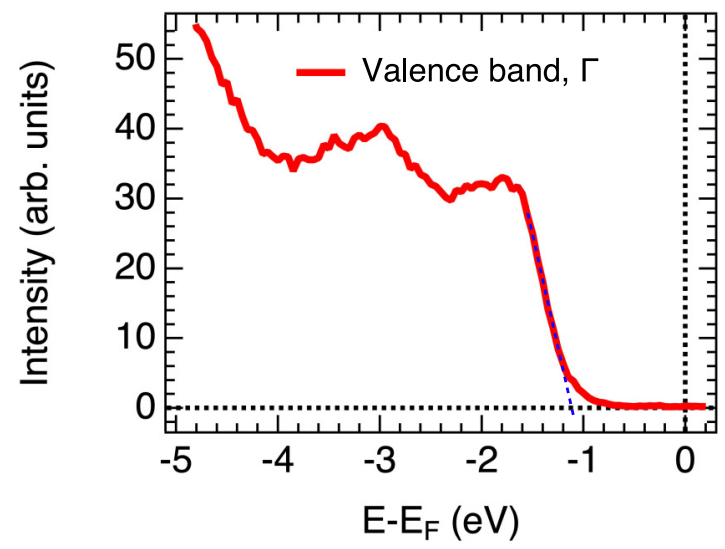

FIG. 2. Valence band spectrum of $\mathrm{Sb}_{2} \mathrm{~S}_{3}$. The valence band spectrum is shown close to the VBM. Data were taken at $\bar{\Gamma}$ with the HHG light source using a photon energy of $15.3 \mathrm{eV}$ and $p$-polarization. Linear extrapolation of the falling edge allows to determine the onset of the VBM (blue dashed line).

are excited from the VB to the $\mathrm{CB}$ where the population is monitored with a $6-\mathrm{eV}$ probe pulse as a function of energy, momentum and pump-probe time delay. Both beams were $p$-polarized with respect to the plane of incidence. The results are summarized in Figs. 3(b), 3(c) and 4. In Fig. 3(b) the energy distribution of electrons after photoexcitation is shown as function of time delay. Additionally, in Fig. 3(c) spectra taken at selected times after excitation are presented. Since the bands disperse little in the vicinity of $\bar{\Gamma}$ the data were integrated in momentum from $0-0.12 \AA^{-1}$ for both plots. Furthermore, the delay-independent background signal of thermally excited electrons was corrected for by subtracting the averaged signal taken at negative time delays where the probe pulse precedes the pump pulse. (The raw data and details to the background correction are given in the Supplemental Material [15]). It can be observed that on a short timescale after photoexcitation higher conduction band states (CBS) are populated. An ultrafast decay within less than $1 \mathrm{ps}$ towards the band minimum is followed on a longer timescale by an accumulation of electrons near the CBM and in a state, which is located in the band gap.

On the spectrum taken at zero delay (black) a continuous band of CBS can be observed with the highest intensity at about $1 \mathrm{eV}$ above the Fermi level. After 0.2 ps (violet spectrum) the highest states have completely vanished and the electrons accumulate in the band minimum resulting in a peak centered at $E-E_{F}=0.7 \mathrm{eV}$. On the spectra at $1 \mathrm{ps}$ and 4 ps (grey and pink spectra, respectively) the peak maximum shifts downwards with increasing time delay to finally reach $E-E_{F}=0.58 \mathrm{eV}$ at $4 \mathrm{ps}$. The time-dependent peak shift and the large peak width indicate the superposition of two states, which are assigned to the CBM and a shallow state in the band gap. The energy positions of these states were determined by fitting the sum of two Gaussians with equal widths to all transient spectra after time zero. The energy positions and widths of both Gaussians were assumed as time independent. (More details are given in the Supplemental Material [15]). Best agreement was obtained for $E-E_{F}=0.64 \mathrm{eV}$ for the CBM and $E-E_{F}=0.58 \mathrm{eV}$ for the shallow gap state. 
(a)

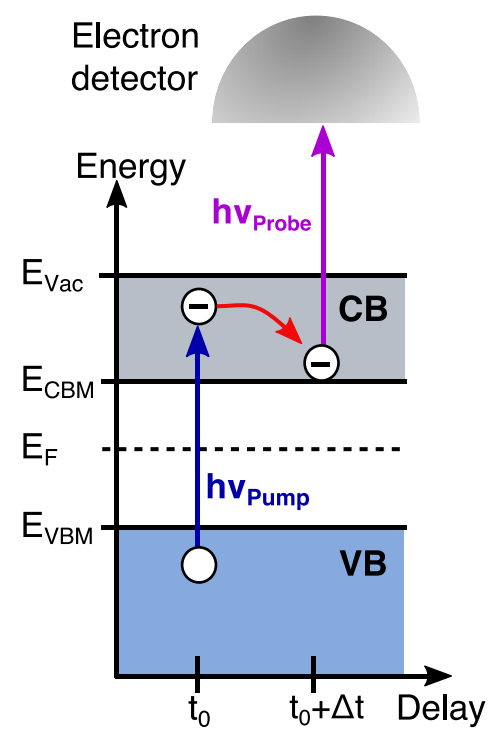

(b)

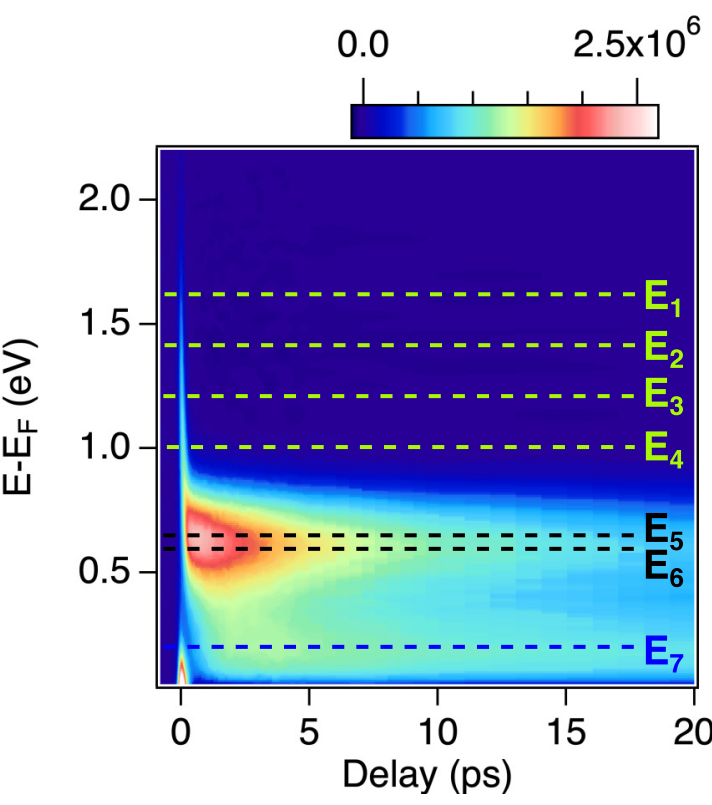

(c)

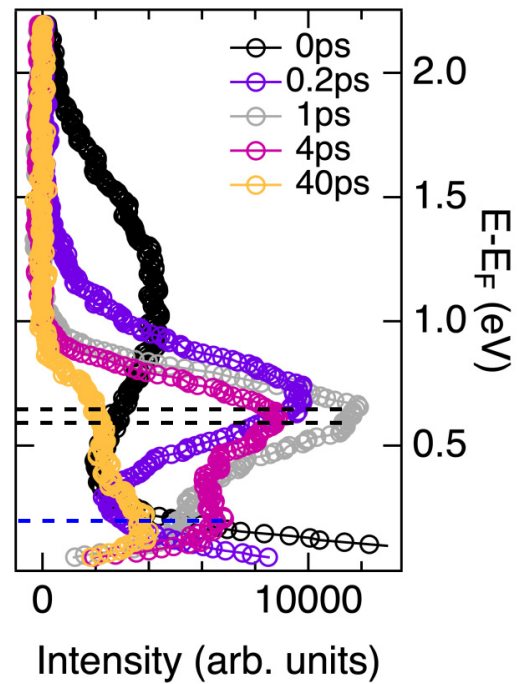

FIG. 3. Time-dependent energy distribution of photoexcited electrons in $\mathrm{Sb}_{2} \mathrm{~S}_{3}$. (a) The principle of tr-2PPE. Absorption of a pump photon leads to the excitation of an electron from the VB to the $\mathrm{CB}$, where the population is probed by absorption of a probe photon as function of time delay. (b) The energy distribution of electrons after photoexcitation with a 3-eV pump pulse is shown color-coded as function of energy and time delay. The energy positions where the charge carrier dynamics were analyzed are indicated. The data were integrated between $0 \AA^{-1}$ and $0.12 \AA^{-1}$. (c) Spectra taken at selected times after photoexcitation. For the data presented in (b,c) the intensity at negative time delays was subtracted from the raw data.

Together with the energy position of the VBM at $E-E_{F}=$ $-1.06 \mathrm{eV}$ the band gap can be determined to be $1.70 \mathrm{eV}$. This value is in good agreement with published experimental $[6,16,19]$ and theoretical [20] values. By comparing our surface-sensitive data or scanning tunneling spectroscopy measurements from Ref. [21] with bulk-sensitive optical transmittance and near-normal reflectance spectra from Ref. [16] no evidence for surface states was found. This is consistent with the fact that the material cleaves between the van der Waals bonded ribbons. Furthermore, on the spectra at longer time delays an additional state appears in the band gap at $E-E_{F}=0.20 \mathrm{eV}$. To investigate the charge carrier dynamics in more detail the time-dependent intensi- ties at different energies $E_{i}$, integrated in momentum within $(0 \pm 0.12) \AA^{-1}$, are shown in Fig. 4. For the CBS the intensities at four arbitrarily chosen equidistant energies $E_{i}$ from 1.6-1.0 eV above the Fermi energy, integrated within $\left(E_{i} \pm 0.05\right) \mathrm{eV}$, were analyzed [Fig. 4(a)]. With decreasing energy an increase in intensity can be observed, accompanied by a shift of the maximum position towards longer time delays and an increasing peak asymmetry on the side of positive time delays where the pump pulse precedes the probe pulse. This shows that the lifetimes of photoexcited charge carriers increase with decreasing energy, with a maximum value of $150 \mathrm{fs}$ measured for these excited electrons. The CBM, which is located at $E-E_{F}=0.64 \mathrm{eV}$, overlaps with a shallow gap (a)

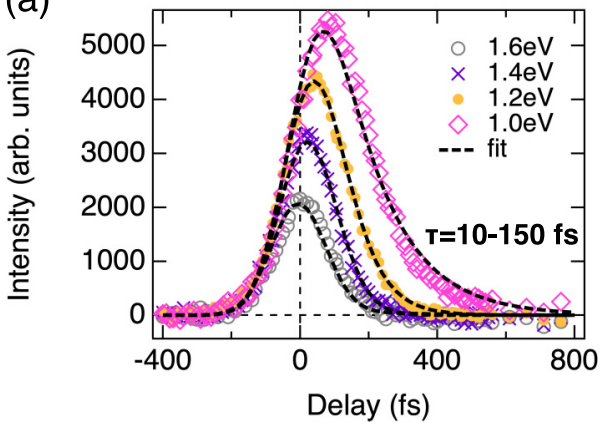

(b)

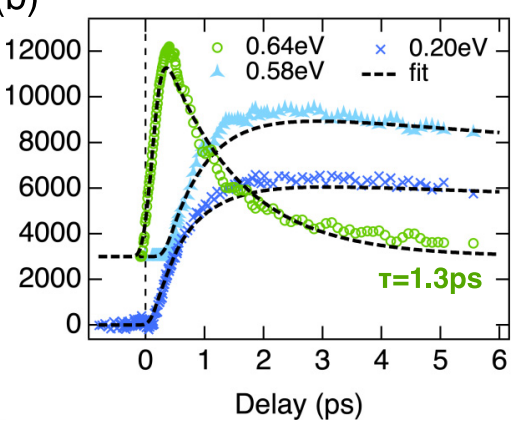

(c)

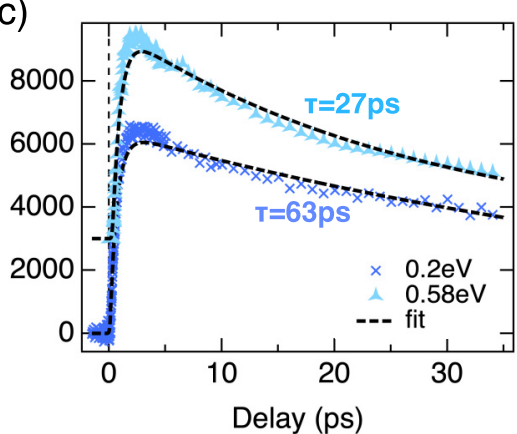

FIG. 4. Relaxation dynamics of photoexcited charge carriers. (a)-(c) Time-dependent electron population at selected energies. Fits based on rate equations are superimposed to the data (black dashed lines). In (b) and (c) the transients at $0.64 \mathrm{eV}$ and $0.58 \mathrm{eV}$ together with the respective fits are scaled by a factor of 0.01 and shifted vertically for better visualization. 
state at $E-E_{F}=0.58 \mathrm{eV}$. The separation of these states was achieved by fitting the sum of two Gaussians centered at the respective energies to all transient spectra after time zero. The time-dependent amplitudes reflect the transient populations in the respective states, which are plotted in Fig. 4(b). Moreover, the time-dependent intensity in the gap state at $E-E_{F}=0.20 \mathrm{eV}$, integrated within $\left(E_{i} \pm 0.05\right) \mathrm{eV}$, is also shown in Figs. 4(b) and 4(c). In the measured raw data the signal of simultaneous two-photon absorption at time zero is seen on top of the transient. This was corrected by subtracting the laser cross correlation from the measured transient. (The raw data and details of the correction procedure are given in the Supplemental Material [15]). For better visualization the transients at $0.64 \mathrm{eV}$ and $0.58 \mathrm{eV}$ were scaled by a factor of 0.01 and shifted vertically. In Fig. 4(b) the population in the CBM increases within the first 0.5 ps followed by a fast depopulation within a few picoseconds. The depletion of the CBM leads to the population of the gap states, which takes about 2 ps before relaxation within a few tens of picoseconds occurs.

The time-dependent populations $N_{i}(t)$ at energies $E_{i}$ can be quantitatively described by a set of rate equations. The energies $E_{i}$ are indicated in Fig. 3(b). By absorption of a Gaussian-shaped pump pulse at $t=t_{0}$ with the temporal width $\sigma_{L}$ an electron population is generated in the intermediate states $i$. Scattering leads to a depopulation which is proportional to the populations $N_{i}(t)$ with characteristic time constants $\tau_{i}$. Furthermore, for lower energies scattering from energetically higher states $k$ into those states $i$ can occur which leads to a time-delayed refilling of energetically lower lying states. The temporal change of the population in the intermediate state $i$ after photoexcitation can be expressed by the following equations:

$$
\begin{aligned}
\frac{d N_{\mathrm{l}}(t)}{d t}= & A_{1} \exp \left\{-\frac{\left(t-t_{0}\right)^{2}}{2 \sigma_{L}^{2}}\right\}-\frac{N_{1}(t)}{\tau_{1}}, \\
\frac{d N_{\mathrm{i}}(t)}{d t}= & A_{i} \exp \left\{-\frac{\left(t-t_{0}\right)^{2}}{2 \sigma_{L}^{2}}\right\}-\frac{N_{\mathrm{i}}(t)}{\tau_{\mathrm{i}}} \\
& +\sum_{k=1}^{i-1} B_{k, i} \frac{N_{\mathrm{k}}(t)}{\tau_{\mathrm{k}}}, \quad(i>1) .
\end{aligned}
$$

Here $A_{i}$ is proportional to the number of electrons excited by the pump pulse into the state $i . B_{k, i}$ denotes the fraction of electrons that decay from an energetically higher-lying state $k<i$ with a lifetime of $\tau_{k}$ into a lower lying state $i$. The measured tr-2PPE signal can be described by a convolution of the Gaussian-shaped probe pulse with the time-dependent populations in the intermediate states $N_{i}(t)$. The cross correlation width of the laser pulses was determined using the transient at $E-E_{F}=1.8 \mathrm{eV}$ with negligible intermediate state lifetime to be 185 fs full width at half maximum. The rate equations were fitted to the experimental transients and the results are superimposed to the measured data in Fig. 4 (all fitting parameters are given in the Supplemental Material [15]).

For the higher conduction bands the populations $N_{i}(t)$ for equidistant states with $i=1, \ldots, 4$ at energies from 1.6-1.0 eV above the Fermi energy were analyzed. Good agreement was obtained without the need to include scattering between those states (all $B_{k, 1-4}=0$ ). For the highest state $E_{1}$ at $1.6 \mathrm{eV}$ a rather short lifetime of $\tau_{1}=10 \mathrm{fs}$ was found. With decreasing energy the lifetime increases up to $\tau_{4}=145$ fs at $1.0 \mathrm{eV}$. As a result, scattering from energetically higher states into states 2 and 3 appears to be negligible, but might contribute to the population of state 4 .

In the $\mathrm{CBM}$ at $E_{5}=0.64 \mathrm{eV}$ a short lifetime of $\tau_{5}=$ 1.3 ps was determined. The occupation of the CBM occurs via photoexcitation by the pump pulse and scattering from the CBS, in particular from $E_{4}\left(B_{4,5} \neq 0\right)$. For the superimposed shallow gap state at energy $E_{6}=0.58 \mathrm{eV}$ a longer lifetime of $\tau_{6}=27 \mathrm{ps}$ was obtained. The occupation of this state is mainly due to scattering from the $\operatorname{CBM}\left(B_{5,6} \neq 0\right)$ and direct population by the pump pulse is negligible $\left(A_{6}=0\right)$. In the gap state at $E_{7}=0.20 \mathrm{eV}$ the longest lifetime of $\tau_{7}=63 \mathrm{ps}$ was determined. As already observed for the shallow gap state at $E_{6}$, the population of this state can be traced back to scattering from the CBM $\left(B_{5,7} \neq 0\right)$ while direct population by the pump pulse can be neglected $\left(A_{7}=0\right)$.

\section{DISCUSSION}

At time zero, energetically high-lying CBS are populated by absorption of pump photons. A fast decay within less than 150 fs into the CBM occurs where a lifetime of $1.3 \mathrm{ps}$ was found. In contrast to other semiconducting photoelectrode materials like $\mathrm{Cu}_{2} \mathrm{O}$ [14] no transport of charge carriers due to band bending is needed to describe the population dynamics. Within the band gap long-lived states were found at $0.06 \mathrm{eV}$ and $0.44 \mathrm{eV}$ below the CBM, reminiscent of defect states. The occupation of those states can be explained by scattering from the CBM rather than by direct photoexcitation.

The charge carrier dynamics in $\mathrm{Sb}_{2} \mathrm{~S}_{3}$ was recently investigated by Yang et al. [13] by means of time-resolved transient absorption and photoluminescence spectroscopy. In this work the authors determined the onset in the absorption spectrum at $1.7 \mathrm{eV}$, which is in agreement with the band-gap value obtained here. In contrast to that, in photoluminescence emission from a state at $1.1 \mathrm{eV}$ is predominant. This energy is close to the value of the gap state found here at $E-E_{\mathrm{VBM}}=1.26 \mathrm{eV}$. Based on intensity-dependent measurements the authors observed that the concentration of those defects exceeds the intrinsic defect concentrations by far. In addition, the light emission from those states on single-crystalline samples is highly polarized. Based on these findings, the authors propose that the respective states are formed by self-trapping and they assign this state to self-trapped excitons (STE).

Self-trapping was considered the first time by Landau in 1933 [22] and is based on strong coupling of free carriers and excitons to phonons. This leads to a localization of the charge carriers by a dense array of phonons due to local deformations of the lattice around the carriers. These deformations generate a potential barrier acting as trap. In systems with at least two dimensions there is an energy barrier that separates free and trapped states and free charge carriers need to pass this barrier by tunneling to become self-trapped. In contrast to that, it was shown by Rashba [23] that in one-dimensional systems like $\mathrm{Sb}_{2} \mathrm{~S}_{3}$ such a potential barrier is not possible and thus, free and self-trapped carriers are in coexistence. In Ref. [24] it was even shown that if the motion of an exciton is limited 


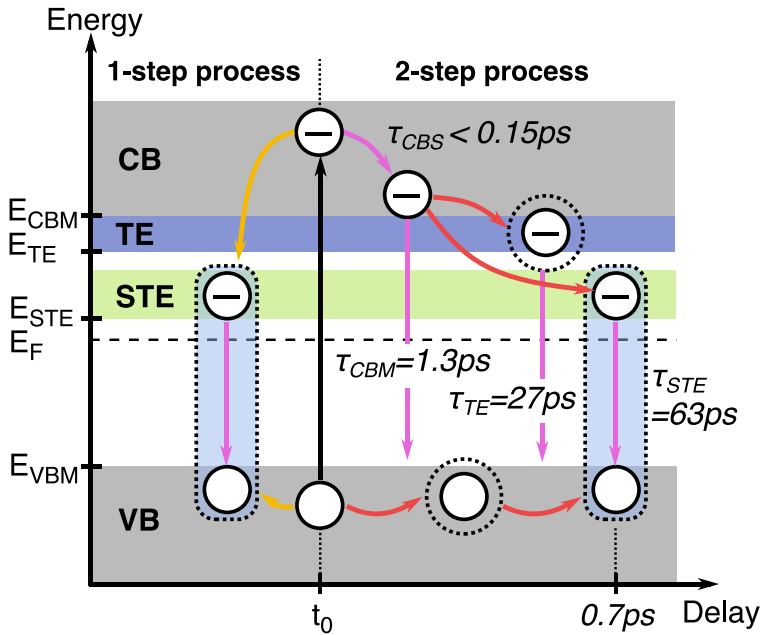

FIG. 5. Relaxation pathways and self-trapping: At $t_{0}$ an electron is excited from the $\mathrm{VB}$ to the $\mathrm{CB}$ due to absorption of a pump photon. In the 1-step process the photogenerated electron-hole pair is immediately trapped by local lattice distortions and forms a STE. In the 2-step process a free electron and hole are trapped separately within the lattice before they merge to form a STE. The trapping of free charge carriers is marked by red and orange arrows, and charge carrier relaxation is indicated by pink arrows. The measured time constants are indicated.

to 1D free excitons are unstable and are always self-trapped. To estimate if this process is likely in $\mathrm{Sb}_{2} \mathrm{~S}_{3}$ in Ref. [13] the elastic constant and Huang Rhys parameter, which is a measure for the electron-phonon coupling strength, are reported. The authors found values for $\mathrm{Sb}_{2} \mathrm{~S}_{3}$, which are indeed close to those of other materials that are known to show self-trapping, like $\mathrm{NaCl}$ or $\mathrm{As}_{2} \mathrm{Se}_{3}$.

Based on this, we propose a model in Fig. 5 that interprets the presented experimental results in the framework of self-trapping. After photoexcitation an electron-hole pair can directly form a STE in a 1-step process. Likewise the formation of the STE may proceed in two steps: first the free electrons and free holes are trapped separately and subsequently the trapped hole captures an electron leading to the formation of a STE. Based on the measured data it is evident that the 2-step process predominates but it cannot be ruled out that also the 1-step process occurs to a lesser extent. The photoexcited electrons relax first towards the CBM within $\tau_{\mathrm{CBS}}<150$ fs. The depopulation of the CBM within $\tau_{\mathrm{CBM}}=1.3 \mathrm{ps}$ can be explained by self-trapping of free carriers. Some of the electrons are trapped by phonons, causing the electrons to slightly lower their energy. The shallow gap state near the CBM is attributed to self-trapping of electrons (TE). Moreover, electrons are captured by trapped holes and form STE. This leads to the formation of the lower-lying gap state. The recombination rates of self-trapped electrons and excitons at the surface are $\tau_{\mathrm{TE}}=27 \mathrm{ps}$ and $\tau_{\mathrm{STE}}=63 \mathrm{ps}$, respectively. The formation time of STE is represented by the rise time of the lower-lying gap state, which is determined by scattering from the CBM. Thus, the STE formation time can be estimated as the time when the decay rate of the CBM is at its maximum, which occurs at 0.7 ps. In Ref. [25] the formation time of an STE in CdSe was determined and a similar value of $0.5 \mathrm{ps}$ is reported.
Molecular dynamics calculations showed that the formation of STE occurs within the period of the lattice vibration [26]. Thus, the frequency of the phonons that trap the exciton can be estimated from the measured formation time of 0.7 ps to be $1.43 \mathrm{THz}$. This value is in good agreement with an optical phonon mode of $\Omega=1.44 \mathrm{THz}$ [27]. The Huang Rhys parameter $S$, which is a measure for the electron-phonon coupling strength, can also be estimated from measured parameters. It can be determined from the energy width $\Delta E$ of the gap state according to [28]

$$
\sqrt{S}=\frac{\Delta E}{2.36 \hbar \Omega \sqrt{\operatorname{coth}\left[(\hbar \Omega) /\left(2 k_{B} T\right)\right]}},
$$

where $k_{B} T$ is the thermal energy of $25 \mathrm{meV}$ at $300 \mathrm{~K}$ and $\hbar$ is the reduced Planck's constant. $\Delta E$ was obtained from a Gaussian fit of the gap state in the spectrum after 40 ps as $267 \mathrm{meV}$ full width at half maximum, which yields $S=41.4$. In addition, $S$ can be determined from the Stokes shift, which corresponds to the energy difference of $0.44 \mathrm{eV}$ between the $\mathrm{CBM}$ and the gap state, by the relations $E_{\mathrm{St}}=2 S \hbar \Omega$ and $E_{\mathrm{St}}=(2 S-1) \hbar \Omega[29]$ as $37.5<S<38$. The estimated values are close to each other and to the value of $S=38.5$, which was published in Ref. [13]. Thus, based on the measured energy shift, the energy width and the formation time of the gap state, self-trapping of excitons by optical phonons with the frequency $\Omega=1.44 \mathrm{THz}$ is proposed.

Based on this analysis, our measurements complement the self-trapping model of Ref. [13]. In this work the Stokes-shift was determined as $0.4 \mathrm{eV}$ and $0.6 \mathrm{eV}$, depending on the growth method and crystallinity of the samples. This agrees well with the value of $0.44 \mathrm{eV}$ obtained in our measurements. The electron-phonon coupling strength of $S=38.5$ reported in Ref. [13] is in agreement with our results where we found $37.5<\mathrm{S}<41.4$. A direct comparison of the lifetimes of selftrapped charge carriers and excitons is not possible since in the time-resolved transient absorption measurements of Ref. [13] the bulk lifetimes are probed whereas tr-2PPE probes the corresponding lifetimes at the surface, which are likely to be shorter due to the presence of additional scattering channels. Qualitatively, the dynamics we measured for the self-trapped excitons agree with the results from Ref. [13]: the formation of STE occurs within a few picoseconds followed by a slow decay on the pico to nanosecond timescale. In Ref. [13] a hole trapping rate of $1.8 \mathrm{ps}$ is reported, which is close to the free carrier lifetime of $1.3 \mathrm{ps}$ found here. The half-life time of STE on polycrystalline samples reported in Ref. [13] is $23 \mathrm{~ns}$, which is larger than the $63 \mathrm{ps}$ determined here. The shorter lifetime measured with tr-2PPE can be traced back to the surface sensitivity of this method. Moreover, in Fig. 4(c) it is recognizable that there are deviations between the measured transient of the STE and the exponential fit since the decay rate of the STE slows down with increasing time delay. Thus, an exponential decay function as used in this work underestimates the lifetime on a longer timescale.

In the following, we compare the charge carrier dynamics in $\mathrm{Sb}_{2} \mathrm{~S}_{3}$ to those observed previously in the iso-electronic compound $\mathrm{Sb}_{2} \mathrm{Se}_{3}$ [30]. Almost identical experimental procedures were used in both cases. In $\mathrm{Sb}_{2} \mathrm{Se}_{3}$ slightly longer lifetimes for the higher conduction bands of 100-300 fs and 
TABLE I. Comparison of $\mathrm{Sb}_{2} \mathrm{~S}_{3}$ and $\mathrm{Sb}_{2} \mathrm{Se}_{3}$. The band gap, energy positions of gap states, lifetimes of charge carriers in the $\mathrm{CB}$, and bulk-to-surface transport are compared at the (100)-surface of $\mathrm{Sb}_{2} \mathrm{~S}_{3}$ and $\mathrm{Sb}_{2} \mathrm{Se}_{3}$. Furthermore, the elastic constants, the Huang Rhys parameters as well as the highest PCE and photovoltages achieved to date are included.

\begin{tabular}{|c|c|c|}
\hline & $\mathrm{Sb}_{2} \mathrm{~S}_{3}$ & $\mathrm{Sb}_{2} \mathrm{Se}_{3}$ \\
\hline \multirow[t]{2}{*}{ Band gap } & $1.7 \mathrm{eV}$ & $1.0 \mathrm{eV}^{\mathrm{a}}$ \\
\hline & Direct, $\Gamma$ & Direct, $\Gamma Z^{\mathrm{a}}$ \\
\hline Gap states $\left(E_{\mathrm{CBM}}-E\right)$ & $0.06 \mathrm{eV}$ (TE), $0.44 \mathrm{eV}$ (STE) & - \\
\hline$\tau_{\mathrm{CBS}}$ & $10-145 \mathrm{fs}$ & $100-300 \mathrm{fs}^{\mathrm{a}}$ \\
\hline \multirow{2}{*}{$\tau_{\mathrm{CBM}}$} & $1.3 \mathrm{ps}$ & 35 ps $(\Gamma Z)^{\mathrm{a}}$ \\
\hline & 27 ps (TE) & 25 ps $(\Gamma Y)^{\mathrm{a}}$ \\
\hline Transport & Not observed & Not observed $^{\mathrm{a}}$ \\
\hline Elastic constant & $38 \mathrm{GPa}^{\mathrm{b}}$ & $34 \mathrm{GPa}^{\mathrm{b}}$ \\
\hline Huang Rhys Parameter & 38 & - \\
\hline PCE and photovoltage & $7.5 \%$ and $0.7 \mathrm{~V}^{\mathrm{c}}$ & $9.2 \%$ and $0.4 \mathrm{~V}^{\mathrm{d}}$ \\
\hline
\end{tabular}

${ }^{\mathrm{a}}$ From Ref. [30].

${ }^{\mathrm{b}}$ From Ref. [33].

${ }^{\mathrm{c}}$ From Ref. [8].

${ }^{\mathrm{d}}$ From Ref. [34].

scattering of electrons between different CBS are reported. However, in $\mathrm{Sb}_{2} \mathrm{~S}_{3}$ the lifetimes are lower with $10-150 \mathrm{fs}$ and we observe direct electron scattering towards the CBM. This can be explained by the smaller band gap of $\mathrm{Sb}_{2} \mathrm{Se}_{3}$ of $1.1 \mathrm{eV}$. Thus, the energy difference between the highest accessible CBS and the band minimum is larger. In $\mathrm{Sb}_{2} \mathrm{~S}_{3}$ a short lifetime of $1.3 \mathrm{ps}$ in the CBM was determined and attributed to trapping of CB electrons in a shallow gap state with a lifetime of $27 \mathrm{ps}$ and to exciton trapping in a lower-lying gap state with a lifetime of $63 \mathrm{ps}$. In comparison, in $\mathrm{Sb}_{2} \mathrm{Se}_{3}$ a decay constant of $35 \mathrm{ps}$ in the CBM is reported, without any evidence for states in the band gap. The missing gap states together with the longer lifetime in the CBM and the single-exponential decay indicate that electrons are not trapped at the surface of $\mathrm{Sb}_{2} \mathrm{Se}_{3}$. In more bulk sensitive fluence- and temperaturedependent terahertz spectroscopy measurements a gap state was observed and, as in $\mathrm{Sb}_{2} \mathrm{~S}_{3}$, the concentration exceeds the defect concentration of the crystal [31,32]. These intrinsically formed gap states can either be explained by self-trapping or by a complex defect structure of such 1D materials owing to the large spaces between the ribbons. In both cases, the missing gap state in tr-2PPE on $\mathrm{Sb}_{2} \mathrm{Se}_{3}$ could be explained by the too shallow probing depth since self-trapping occurs in the bulk of a crystal and the STE might decay when it reaches the surface. In Table I the properties of $\mathrm{Sb}_{2} \mathrm{~S}_{3}$ and $\mathrm{Sb}_{2} \mathrm{Se}_{3}$ are compared.

As photoelectrode material in solar devices both compounds show comparatively low efficiencies and photovoltages combined with poor transport properties. The highest values reached so far are $7.5 \%$ and $0.7 \mathrm{~V}$ for $\mathrm{Sb}_{2} \mathrm{~S}_{3}$ [8] and $9.2 \%$ and $0.4 \mathrm{~V}$ for $\mathrm{Sb}_{2} \mathrm{Se}_{3}$ [34], respectively. In the present and recently published Ref. [30] tr-2PPE measurements a long-living population at higher energies at the surface was found in both materials. Therefore, it is proposed that the losses can be attributed to a low charge carrier mobility due to trapping. For both materials it was reported that the concentration of gap states detected in measurements exceeds the defect concentrations of the crystals by several orders of magnitude. This leads to the conclusion that in these 1D materials trap states are formed intrinsically. Despite some ambiguities in the case of $\mathrm{Sb}_{2} \mathrm{Se}_{3}$, in $\mathrm{Sb}_{2} \mathrm{~S}_{3}$ all experimental observations can be well explained in the framework of self-trapping. In particular, the presence of free and trapped electrons in the CBM and the appearance of a gap state fit well into the picture of self-trapping. The corresponding Stokes shift, the energy width and the formation time agree with the values that are expected for self-trapping by $1.44 \mathrm{THz}$ optical phonons. These experimental observations are barely explainable by conventional defect trapping. As a consequence, to increase the PCE of $\mathrm{Sb}_{2} \mathrm{~S}_{3}$ solar devices, self-trapping should be avoided, e.g., by stiffening the elastic properties by strain in order to increase the energy cost for the formation of local lattice distortions.

The supporting data for this article are available via Zenodo [35].

\section{ACKNOWLEDGMENTS}

The research was funded by the Swiss National Science Foundation under Grant No. 200020_172641 and by the University of Zurich through the Research Priority Project LightChEC.

J.O. and M.H. conceived the experiments, L.G. conducted the experiments and analyzed the results, and F.O.v.R. prepared the samples. L.G. wrote the manuscript and all authors reviewed it.

The authors declare no competing interests.
[1] N. W. Tideswell, F. H. Kruse, and J. D. McCullough, The crystal structure of antimony selenide, $\mathrm{Sb}_{2} \mathrm{Se}_{3}$, Acta Crystallogr. 10, 99 (1957).
[2] P. Bayliss and W. Nowacki, Refinement of the crystal structure of stibnite, $\mathrm{Sb}_{2} \mathrm{~S}_{3}$, Z. Kristallogr. Crystalline Materials 135, 308 (1972). 
[3] Y. Zhou, L. Wang, S. Chen, S. Qin, X. Liu, J. Chen, D.-J. Xue, M. Luo, Y. Cao, Y. Cheng, E. H. Sargent, and J. Tang, Thin-film $\mathrm{Sb}_{2} \mathrm{Se}_{3}$ photovoltaics with oriented one-dimensional ribbons and benign grain boundaries, Nat. Photon. 9, 409 (2015).

[4] C. Gao, M. Xu, B. Ng, L. Kang, L.-X. Jiang, Y. Lai, and F. Liu, In situ growth of $\mathrm{Sb}_{2} \mathrm{~S}_{3}$ thin films by reactive sputtering on $\mathrm{n}-\mathrm{Si}$ (100) substrates for top sub-cell of silicon based tandem solar cells, Mater. Lett. 195, 186 (2017).

[5] R. R. Prabhakar, T. Moehl, S. Siol, J. Suh, and S. D. Tilley, $\mathrm{Sb}_{2} \mathrm{~S}_{3} / \mathrm{TiO}_{2}$ heterojunction photocathodes: Band alignment and water splitting properties, Chem. Mater. 32, 7247 (2020).

[6] Y.-C. Wang, Y.-Y. Zeng, L.-H. Li, C. Qin, Y.-W. Wang, Z.-R. Lou, F.-Y. Liu, Z.-Z. Ye, and L.-P. Zhu, A stable and efficient photocathode using an $\mathrm{Sb}_{2} \mathrm{~S}_{3}$ absorber in a near-neutral electrolyte for water splitting, ACS Appl. Energy Mater. 3, 6188 (2020).

[7] A. D. Vos, Detailed balance limit of the efficiency of tandem solar cells, J. Phys. D 13, 839 (1980).

[8] Y. C. Choi, D. U. Lee, J. H. Noh, E. K. Kim, and S. I. Seok, Highly improved $\mathrm{Sb}_{2} \mathrm{~S}_{3}$ sensitized-inorganic-organic heterojunction solar cells and quantification of traps by deep-level transient spectroscopy, Adv. Funct. Mater. 24, 3587 (2014).

[9] C. Jiang, R. Tang, X. Wang, H. Ju, G. Chen, and T. Chen, Alkali metals doping for high-performance planar heterojunction $\mathrm{Sb}_{2} \mathrm{~S}_{3}$ solar cells, Solar RRL 3, 1800272 (2019).

[10] S. Rühle, Tabulated values of the Shockley-Queisser limit for single junction solar cells, Solar Energy 130, 139 (2016).

[11] P. Büttner, F. Scheler, C. Pointer, D. Döhler, M. K. S. Barr, A. Koroleva, D. Pankin, R. Hatada, S. Flege, A. Manshina, E. R. Young, I. Mínguez-Bacho, and J. Bachmann, Adjusting interfacial chemistry and electronic properties of photovoltaics based on a highly pure $\mathrm{Sb}_{2} \mathrm{~S}_{3}$ absorber by atomic layer deposition, ACS Appl. Energy Mater. 2, 8747 (2019).

[12] J. A. Christians and P. V. Kamat, Trap and transfer. Two-step hole injection across the $\mathrm{Sb}_{2} \mathrm{~S}_{3} / \mathrm{CuSCN}$ interface in solid-state solar cells, ACS Nano 7, 7967 (2013).

[13] Z. Yang, X. Wang, Y. Chen, Z. Zheng, Z. Chen, W. Xu, W. Liu, Y. M. Yang, J. Zhao, T. Chen, and H. Zhu, Ultrafast self-trapping of photoexcited carriers sets the upper limit on antimony trisulfide photovoltaic devices, Nat. Commun. 10, 4540 (2019).

[14] L. Grad, Z. Novotny, M. Hengsberger, and J. Osterwalder, Influence of surface defect density on the ultrafast hot carrier relaxation and transport in $\mathrm{Cu}_{2} \mathrm{O}$ photoelectrodes, Sci. Rep. 10, 10686 (2020).

[15] See Supplemental Material at http://link.aps.org/supplemental/ 10.1103/PhysRevMaterials.5.075401 for the raw data and details of the correction procedure.

[16] M. Y. Versavel and J. A. Haber, Structural and optical properties of amorphous and crystalline antimony sulfide thin-films, Thin Solid Films 515, 7171 (2007).

[17] C. Monney, A. Schuler, T. Jaouen, M.-L. Mottas, T. Wolf, M. Merz, M. Muntwiler, L. Castiglioni, P. Aebi, F. Weber, and M. Hengsberger, Robustness of the charge-ordered phases in $\mathrm{IrTe}_{2}$ against photoexcitation, Phys. Rev. B 97, 075110 (2018).

[18] A. C. Schuler, Design and implementation of a monochromatic high harmonic generation light source at $100 \mathrm{kHz}$ and its application to time- and angle-resolved photoelectron spectroscopy on $\mathrm{Bi}_{2} \mathrm{Se}_{3}$, Ph.D. thesis, University of Zurich, Zürich, 2018 .
[19] C. Diliegros Godines, J. Santos, N. Mathews, and M. Pal, Effect of Ag doping on structural, optical and electrical properties of antimony sulfide thin films, J. Mater. Sci. 53, 11562 (2018).

[20] T. Fujita, K. Kurita, K. Takiyama, and T. Oda, The fundamental absorption edge and electronic structure in $\mathrm{Sb}_{2} \mathrm{~S}_{3}$, J. Phys. Soc. Jpn. 56, 3734 (1987).

[21] A. Maiti, S. Chatterjee, and A. J. Pal, Sulfur-vacancy passivation in solution-processed $\mathrm{Sb}_{2} \mathrm{~S}_{3}$ thin films: Influence on photovoltaic interfaces, ACS Appl. Energy Mater. 3, 810 (2020).

[22] L. D. Landau, Electron motion in crystal lattices, Phys. Z. Sowjet. 3, 664 (1933).

[23] E. I. Rashba, Progress in exciton spectroscopy: Personal perspective, J. Lumin. 87-89, 1 (2000).

[24] H. Sumi and A. Sumi, Dimensionality dependence in selftrapping of excitons, J. Phys. Soc. Jpn. 63, 637 (1994).

[25] X. Sui, X. Gao, X. Wu, C. Li, X. Yang, W. Du, Z. Ding, S. Jin, K. Wu, T. C. Sum, P. Gao, J. Liu, X. Wei, J. Zhang, Q. Zhang, Z. Tang, and X. Liu, Zone-folded longitudinal acoustic phonons driving self-trapped state emission in colloidal CdSe nanoplatelet superlattices, Nano Lett. 21, 4137 (2021).

[26] K. Ishida, Self-trapping dynamics of excitons on a onedimensional lattice, Z. Phys. B 102, 483 (1997).

[27] Antimony sulfide $\left(\mathrm{Sb}_{2} \mathrm{~S}_{3}\right)$ phonon frequencies: Datasheet from Landolt-Börnstein - Group III Condensed Matter Volume 41C: "Non-Tetrahedrally Bonded Elements and Binary Compounds I” in SpringerMaterials, edited by O. Madelung, U. Rössler, and M. Schulz (Springer-Verlag, Berlin, 1998).

[28] W. Stadler, D. M. Hofmann, H. C. Alt, T. Muschik, B. K. Meyer, E. Weigel, G. Müller-Vogt, M. Salk, E. Rupp, and K. W. Benz, Optical investigations of defects in $\mathrm{Cd}_{1-x} \mathrm{Zn}_{x} \mathrm{Te}$, Phys. Rev. B 51, 10619 (1995).

[29] M. de Jong, L. Seijo, A. Meijerink, and F. T. Rabouw, Resolving the ambiguity in the relation between Stokes shift and HuangRhys parameter, Phys. Chem. Chem. Phys. 17, 16959 (2015).

[30] L. Grad, F. von Rohr, J. Zhao, M. Hengsberger, and J. Osterwalder, Photoexcited charge carrier dynamics in $\mathrm{Sb}_{2} \mathrm{Se}_{3}$ (100), Phys. Rev. Materials 4, 105404 (2020).

[31] K. Wang, C. Chen, H. Liao, S. Wang, J. Tang, M. C. Beard, and Y. Yang, Both free and trapped carriers contribute to photocurrent of $\mathrm{Sb}_{2} \mathrm{Se}_{3}$ solar cells, J. Phys. Chem. Lett. 10, 4881 (2019).

[32] W. Yang, S. Lee, H.-C. Kwon, J. Tan, H. Lee, J. Park, Y. Oh, H. Choi, and J. Moon, Time-resolved observations of photogenerated charge-carrier dynamics in $\mathrm{Sb}_{2} \mathrm{Se}_{3}$ photocathodes for photoelectrochemical water splitting, ACS Nano 12, 11088 (2018).

[33] H. Koc, A. M. Mamedov, E. Deligoz, and H. Ozisik, First principles prediction of the elastic, electronic, and optical properties of $\mathrm{Sb}_{2} \mathrm{~S}_{3}$ and $\mathrm{Sb}_{2} \mathrm{Se}_{3}$ compounds, Solid State Sci. 14, 1211 (2012).

[34] Z. Li, X. Liang, G. Li, H. Liu, H. Zhang, J. Guo, J. Chen, K. Shen, X. San, W. Yu, R. E. I. Schropp, and Y. Mai, 9.2\%efficient core-shell structured antimony selenide nanorod array solar cells, Nat. Commun. 10, 125 (2019).

[35] L. Grad, F. O. von Rohr, M. Hengsberger, and J. Osterwalder, Data for "Charge carrier dynamics and self-trapping on $\mathrm{Sb}_{2} \mathrm{~S}_{3}$ (100)," https://doi.org/10.5281/zenodo.6840656 (2021).

Correction: The data availability statement was missing and has been inserted. 